\title{
Studies of a glow discharge electron beam
}

\author{
Zeng qi $\mathrm{Yu}^{\text {a) }}{ }^{\text {Jorge J. Rocca, }}{ }^{\text {b) }}$ and George J. Collins ${ }^{c)}$ \\ Electrical Engineering Department, Colorado State University, Fort Collins, Colorado 80523
}

(Received 26 April 1982; accepted for publication 14 May 1982)

The energy spectrum of a kilovolt electron beam, generated by a helium glow discharge at pressures between 0.15 and 0.8 Torr, was measured with an electrostatic energy analyzer. An abrupt increase in the energy spread of the electron beam was observed to be coincident with an increase in the luminosity of the electron beam created plasma, and with the onset of intense microwave radiation. The relevance of these phenomena in cw electron beam pumped ion lasers is discussed.

PACS numbers: $52.80 . \mathrm{Hc}, 41.80 . \mathrm{Dd}, 52.40 . \mathrm{Mj}$

\section{INTRODUCTION}

Under proper conditions a glow discharge may produce powerful electron beams. Glow discharge electron beams can be generated in helium at pressures from 0.1 to 2 Torr without differential pumping. ${ }^{l, 2}$ In contrast, the high-voltage operation of a hot cathode electron gun requires ambient pressures below $10^{-4}$ Torr. We have used glow discharge created electron beams at $1.5-3 \mathrm{keV}$ and currents up to 1 amp to pump cw ion lasers. We have obtained cw laser action in both singly ionized mercury and iodine in heliummetal vapor mixtures. ${ }^{3,4}$ Electron beam pumping is a new laser excitation scheme that could improve both the output power and efficiency of ion lasers due to the large density of energetic electrons in the active plasma medium as compared to conventional schemes. ${ }^{5}$

The energy spectrum of electrons emitted by a glow discharge has been measured previously ${ }^{6}$ at discharge currents of a few milliamps with a cathode current density of up to $0.40 \mathrm{~mA} / \mathrm{cm}^{2}$. We have measured the electron beam energy distribution at currents between 20 and $700 \mathrm{~mA}$, and helium pressures between 0.15 and 0.8 Torr using an electrostatic energy analyzer. Our measurements correspond to cathode current densities from 2 to $70 \mathrm{~mA} / \mathrm{cm}^{2}$. We observed that at a critical electron beam current density, dependent on pressure, a region of the electron-beam-generated plasma with unusually high luminosity was produced. The onset of this phenomena was found to be coincident with an abrupt degradation of the electron beam energy profile. We demonstrated that the onset of intense optical emission from the plasma was correlated with a beam-plasma interaction that also produces ultrahigh frequency (UHF) radiation.

\section{GLOW DISCHARGE CREATED ELECTRON BEAM}

The electron gun consists of a cylindrical magnesium cathode $3.5 \mathrm{~cm}$ in diameter surrounded by a ceramic $\left(\mathrm{Al}_{2} \mathrm{O}_{3}\right)$ tube that confines electron emission to the cathode front face. The distance between the cathode and the ceramic shield is approximately $1 \mathrm{~mm}$. The front face of the cathode is concave with a $9 \mathrm{~cm}$ radius of curvature to obtain a self-

\footnotetext{
a) Permanent address: Department of Physics, Fudan University, Shanghai, China.

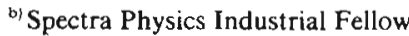

${ }^{c}$ Alfred Sloan Fellow 1979-1982.
}

focused electron beam. Cathode curvature shapes the electric field in the cathode fall region so that a converging electron beam results. This electron beam, with the focus between the cathode and a target, is shown in Fig. 1(a). The cathode is water cooled to allow direct current operation at large discharge powers $(\geqslant 1 \mathrm{~kW})$. The position of the anode is not important and does not influence the electron beam as long as the anode position is outside the glow discharge dark space.

Secondary electron emission from the cathode ${ }^{7}$ occurs following bombardment of the cathode by ions which are accelerated through the cathode dark space, where practi-
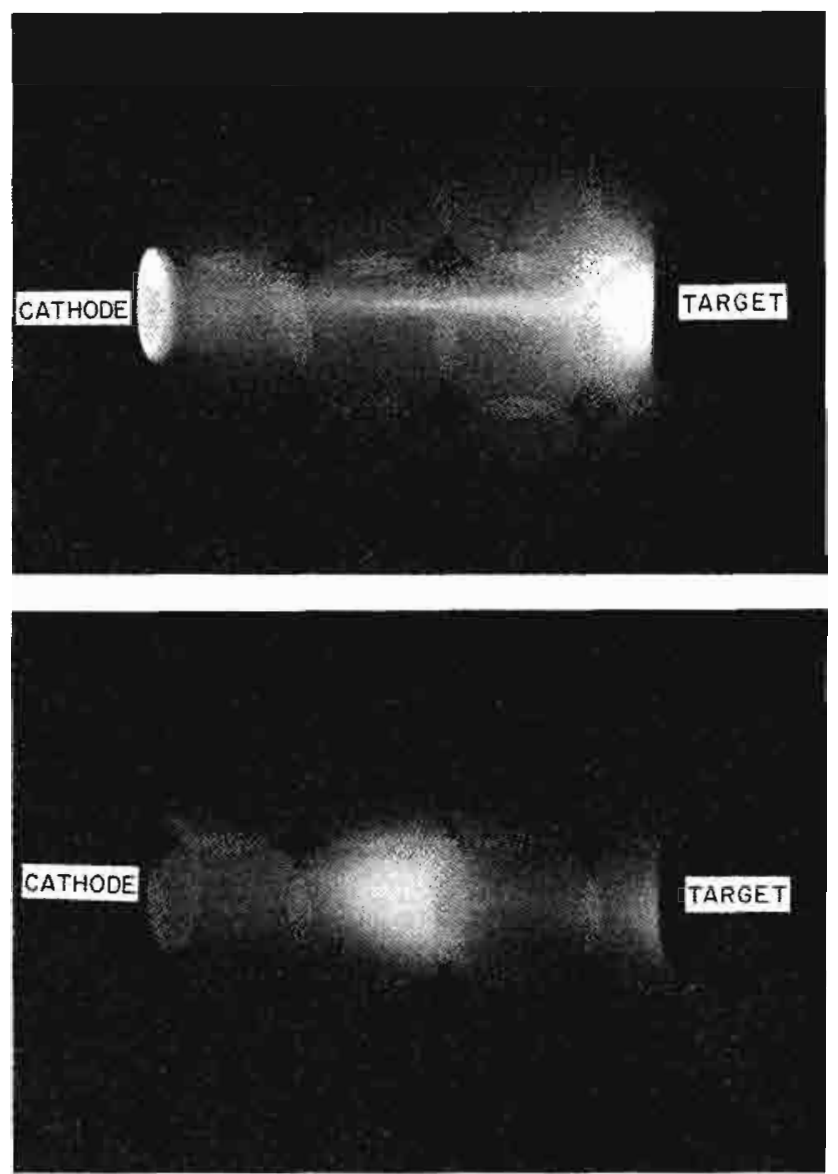

FIG. 1 (a) Self-focused electron beam discharge. (b) Electron beam discharge showing the high luminosity plasma that appears in the focal region of the electron beam in between the cathode and the target. 
cally all the discharge voltage drops occur. Energetic neutrals, created by resonant charge transfer collisions ${ }^{8}$ in the cathode fall region, also contribute to electron emission. The electrons emitted from the cathode surface are also accelerated in the dark space, in the opposite direction, to form the electron beam.

In preliminary studies with $\mathbf{M g}$ cathodes, we observed that the secondary electron emission decreased by more than an order of magnitude as the native magnesium oxide was removed from the cathode surface with ion sputtering. We have determined that a large secondary electron emission from the cathode can be maintained by the addition of a small amount of oxygen (10-20 mTorr) to the discharge chamber which enables the maintenance of a magnesium oxide coating on the cathode surface. This oxide coating permits the production of the electron beam with high efficiency while at the same time reducing cathode sputtering. Using a calorimeter to measure the electron beam power directly, we found that up to $70 \%$ of the discharge power goes into the electron beam when the discharge voltage is $2.4 \mathrm{kV}$. Neglecting ionization in the dark space the electron beam current, $I_{e}$, is related to the ion current, $I_{+}$, by the relationship $I_{e}=\gamma I_{+}$, where $\gamma$ is the secondary electron emission coefficient. Considering that $I=I_{e}+I_{+}$, we can state

$$
I_{e}=\frac{\gamma}{1+\gamma} I \text {. }
$$

Hence, from the calorimetric measurement of the electron beam power an effective secondary emission coefficient $\gamma$ of 2.5 is obtained for the magnesium cathode with an oxide coating from Eq. (1).

\section{ELECTRON BEAM ENERGY SPECTRUM MEASUREMENTS}

The electron beam energy spectrum was measured with an electrostatic energy analyzer, using the experimental setup shown in Fig. 2. The energy analyzer chamber is connected to the electron beam glow discharge housing through a $0.05-\mathrm{mm}$-diam sampling hole. The pinhole was placed $17 \mathrm{~cm}$ from the cathode emitting surface. The wall dividing both chambers is made of copper and is water cooled in order to withstand the impinging electron beam which delivers several hundred watts. The electron analyzer chamber is pumped with a turbomolecular pump and the pressure is maintained below $10^{-5}$ Torr. Helium is flowed through the electron beam glow discharge chamber, via a needle valve, and pumped out by a rotary vacuum pump. A small amount of oxygen, accounting for a partial pressure of 10-20 m Torr in the discharge chamber, was also flowed for increased electron emission as described in Sec. II. The presence of oxygen at these partial pressures was shown to have no significant influence on the electron energy profiles.

The electron gun was mounted in a micropositioner that allows alignment of the electron beam both with the sampling hole and the entrance hole of the energy analyzer. The electrostatic energy analyzer used was a Comstock, Inc., Model AC- 901 with $160^{\circ}$ spherical sector surfaces providing an energy resolution of $0.5 \%$ with a $1-\mathrm{mm}$-diam entrance aperture to the energy analyzer. ${ }^{9}$ Electron beam energy distributions were measured at $0.15,0.2,0.4,0.6$, and 0.8 Torr of helium.

Illustrative data obtained from measurements at 0.4 ,

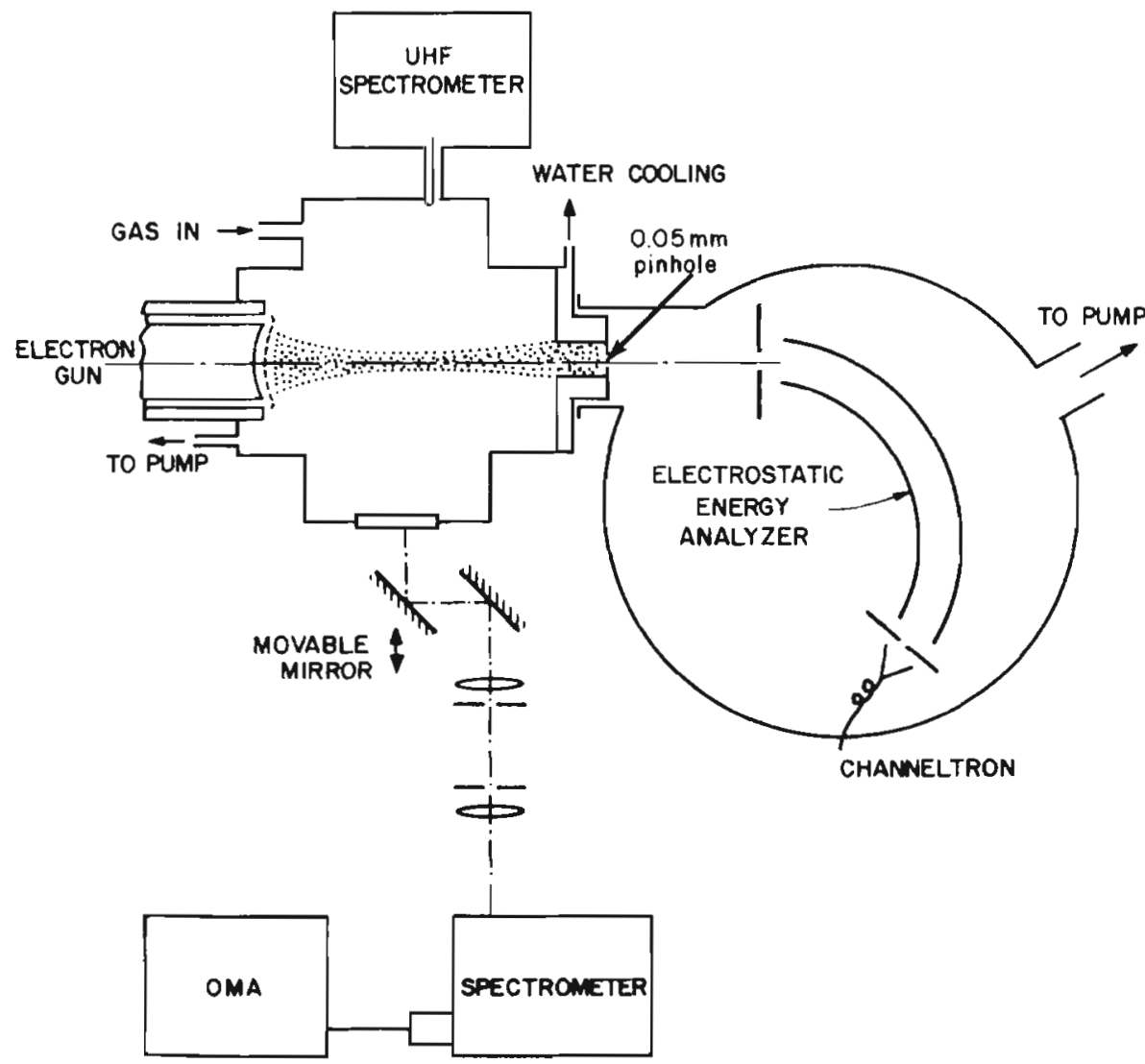

FIG. 2. Experimental setup used to study the electron beam glow discharge. 


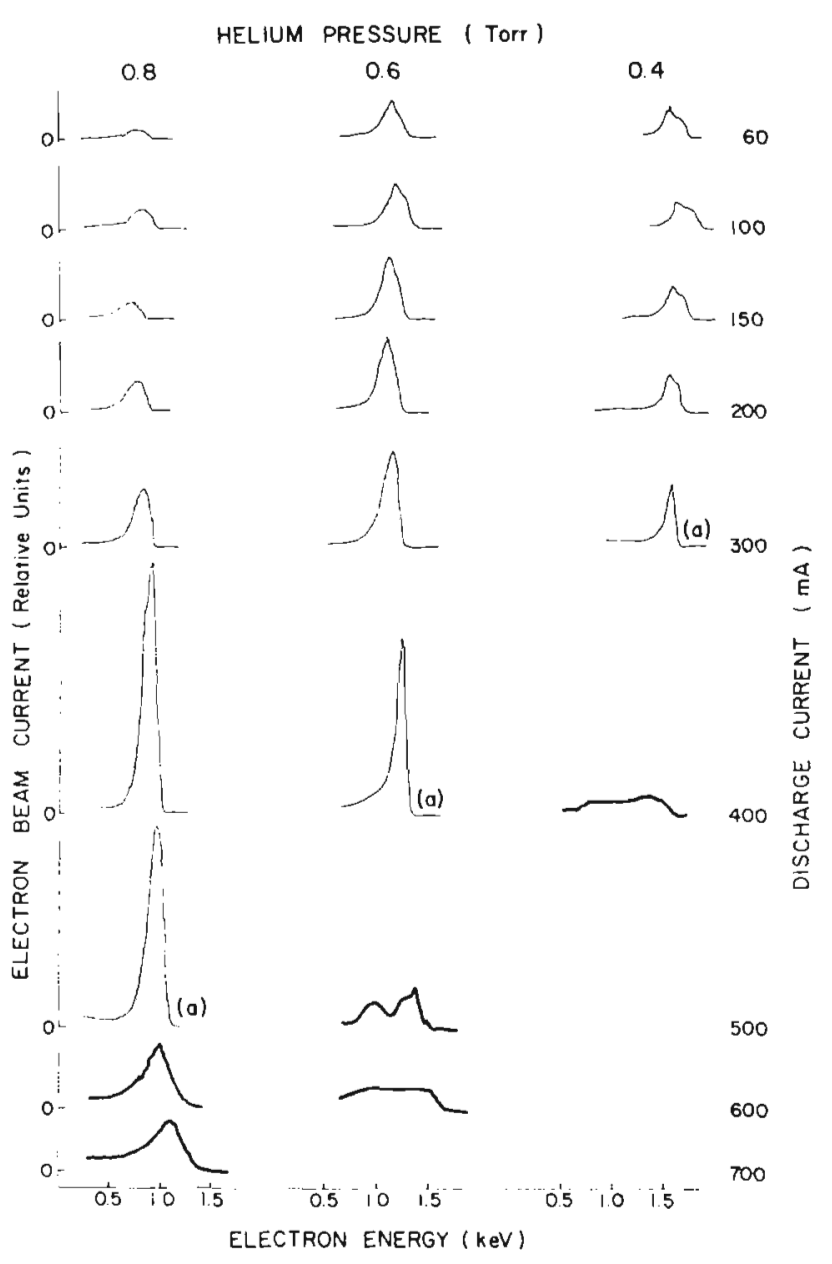

FIG. 3. Measured electron beam energy spectrums at $0.4,0.6$, and 0.8 Torr of helium are shown in three columns. The discharge current corresponding to each row is shown on the right side of the figure.

0.6, and 0.8 Torr are shown in three columns in Fig. 3 for currents between 60 and $700 \mathrm{~mA}$. The individual plots show the measured energy spectrum of the electron beam at nine different discharge currents indicated on the right-hand side of Fig. 3. Note that the zero of electron beam current for each energy spectrum is indicated on the left of the figure and the energy axis at the bottom. The same relative scale was used to plot the electron beam current for every spectrum in Fig. 3. With data in this format the following trends can be observed. For all pressures the peak value of the electron beam current increases as the current is incremented, up to a critical value denoted as (a) in the series of energy spectrums of Fig. 3. Beyond this critical current, the electron beam energy profile, that up to this point was getting narrower (typically $100-300 \mathrm{eV}$ full width at half-maximum) abruptly degrades into a broad profile as shown. This change in the electron beam energy spectrum is associated with the sudden appearance of a plasma region with very intense luminosity, close to the focus of the electron beam. Figure 1(b) shows the electron beam glow discharge with the presence of a high luminosity plasma near the focus of the electron beam. This figure corresponds to the degraded electron beam profiles denoted in Fig. 3 by thick lines. In Fig. 1(a), the electron beam is well collimated and corresponds to the elec-

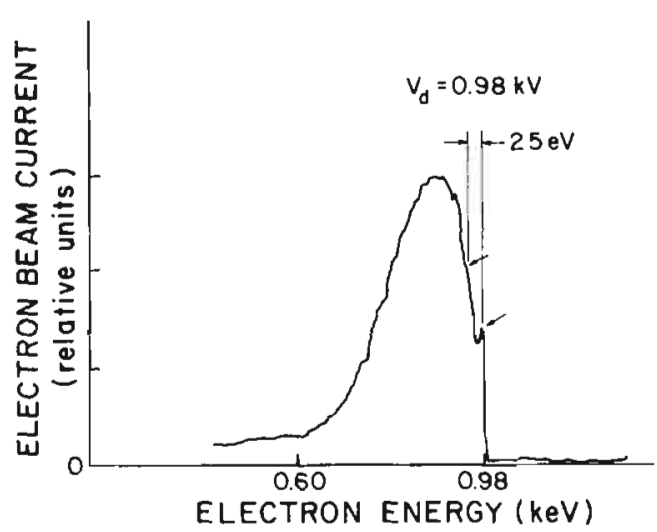

FIG. 4. Electron beam energy spectrum measured at 0.8 Torr of helium and at $0.3 \mathrm{~A}$ discharge current.

tron energy profiles in Fig. 3 denoted by narrow lines.

Figure 4 shows one illustrative electron beam energy spectrum measured at 0.8 Torr of $\mathrm{He}$ and at $0.3 \mathrm{~A}$ discharge current. This spectrum corresponds to the spectrum in the fifth row of column one in Fig. 3. Several peaks are observed in this spectrum, at intervals of approximately $25 \mathrm{eV}$, which coincides with the ionization energy of helium $(24.8 \mathrm{eV})$. Beam electrons that suffered one, two, or more ionizing collisions constitute the different peaks. Elastic electron-helium collisions smooth out the distribution. The important observation to be made in Fig. 4 is that the maximum measured electron beam energy corresponds to the measured discharge voltage, $V_{d}$. This confirms that nearly all the discharge voltage drops in the cathode fall region, where the electrons are accelerated.

\section{OPTICAL MEASUREMENTS}

The light emitted by the electron-beam-created glow discharge was monitored using an optical multichannel analyzer (OMA) having an intensified diode array detector of 512 elements. The optical setup shown in Fig. 2 was used. The detector was mounted on a $1 / 2$ meter spectrometer having a diffraction grating of 280 groves per millimeter. This provided a resolution of $6 \AA$ per channel and a spectral range of nearly $3000 \AA$. We were also able to make spatially resolved ( $1 \mathrm{~mm}$ resolution) measurements in different regions of the glow discharge, shown in Fig. 1 (b) by moving one of the two parallel mirrors shown. Special attention was given to the focal region of the electron beam, in the negative glow region of the discharge, where the unusually high luminosity shown in Fig. 1(b) suddenly appears.

Most of the visible radiation from the electron beam helium discharge was observed to be from three $\mathrm{He} I$ spectral lines: $5015.7 \AA\left(3 p^{1} P^{0} \rightarrow 2 s^{1} S\right), 5875.6 \AA\left(3 d^{3} D_{2} \rightarrow 2 p^{3} P^{0}\right)$, and $6678.1 \AA\left(3 d^{1} D \rightarrow 2 p^{1} P^{0}\right)$. The intensity of these lines in the focal region of the electron beam as a function of discharge current is shown in Fig. 5. If single step electron excitation from the helium atom ground state is the major creation mechanism, the intensity of the $5015.7 \AA$ lines should dominate over the other two lines, since the $3^{1} P$ level has the largest electron impact excitation cross section. ${ }^{10}$ This is the case observed at low discharge currents when the negative 


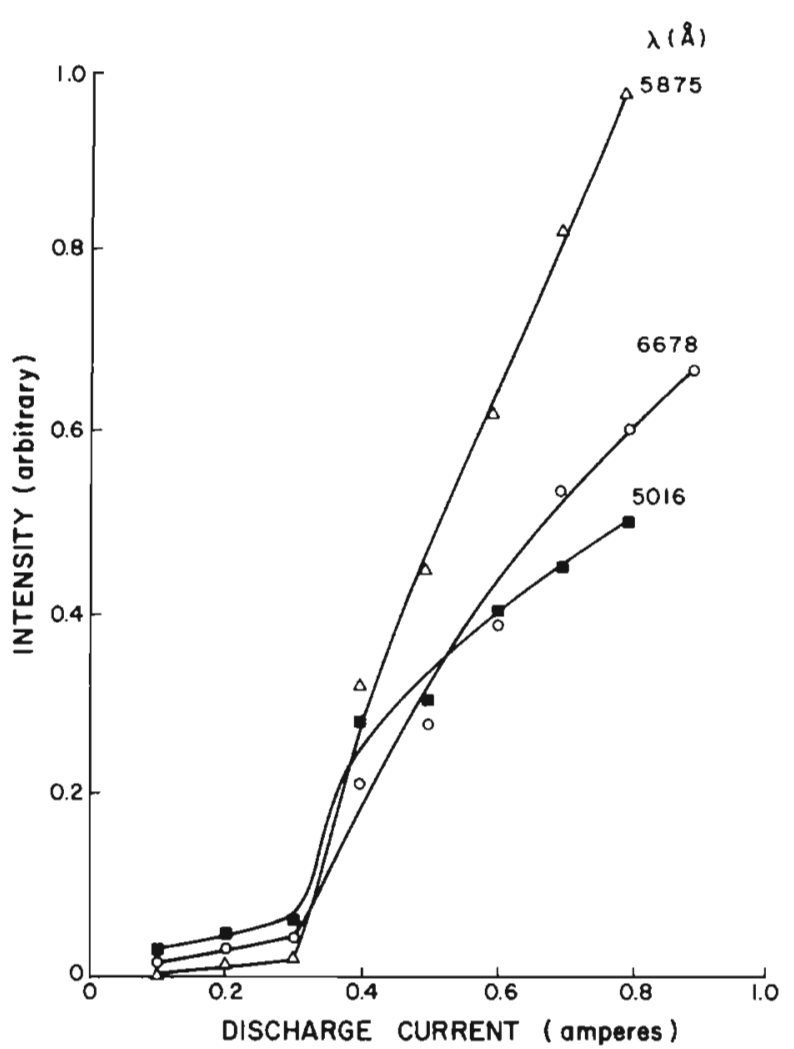

FIG. 5. Behavior of the spontaneous emissions originating from the focal region of the electron beam helium discharge. Helium pressure was 0.2 Torr.

glow, as observed by the eye, is dominantly green. In this low current range the intensity of the lines increases roughly linearly with beam current up to a critical current. Then an abrupt increase in the luminosity of this plasma region occurs, as shown in Fig. 5. When this occurs the plasma looks as shown in Fig. 1(b). Now emission from the 5875.6 line dominates, making the region of the plasma appear pink/ orange to the eye. A change occurs in the electron energy distribution as shown in spectrum drawn with thick lines in

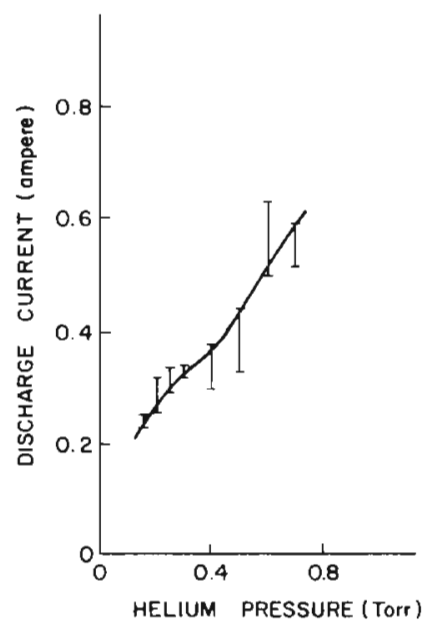

FIG. 6. Current onset of the plasma "ball of light" as a function of helium pressure. The high luminosity plasma region is observed for the conditions above the curve.
Fig. 3, and direct excitation from the helium ground state by beam electrons is no longer the dominant excitation mechanism. The discharge current at which the sudden change in plasma luminosity occurs increases as the helium pressure is incremented. This is shown in Fig. 6. Below we describe the origin of the change in the electron beam energy spectrum and the correlation with the appearance of a luminous region in the beam-generated plasma.

\section{ULTRAHIGH FREQUENCY (UHF) GENERATION}

The degradation in the electron beam energy profile and the observation of an abrupt increase in the intensity of the visible radiation emitted by the discharge previously described suggests the existence of strong beam plasma interactions. ${ }^{1-13}$ A confirmation of the existence of plasma oscillations excited by the electron beam is the observation of an abrupt increase in the intensity of the microwave radiation generated by the discharge, in coincidence with the appearance of the optical emission phenomena described above. Figure 7 shows the $X$ band UHF emission intensity as a function of the electron beam discharge current. The beam current at which the UHF radiation abruptly increases was always in coincidence with the appearance of the "ball of light" in the plasma region [shown in Fig. 1(b)] and with the pronounced degradation of the electron beam energy spectrum. Specifically, in Fig. 3 for a pressure of 0.6 Torr the onset of beam degradation occurs at $500 \mathrm{~mA}$, which agrees with the current for intense UHF oscillation shown in Fig. 7. The same agreement between beam degradation and UHF threshold occurs at $0.4 \mathrm{~A}$ with a helium pressure of 0.4 Torr.

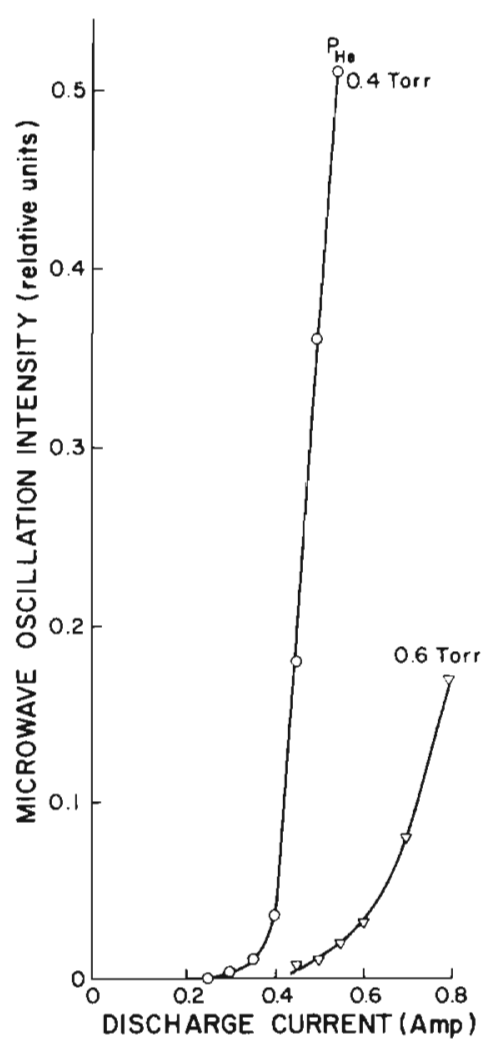

FIG. 7. Intensity of microwave radiation as a function of discharge current 


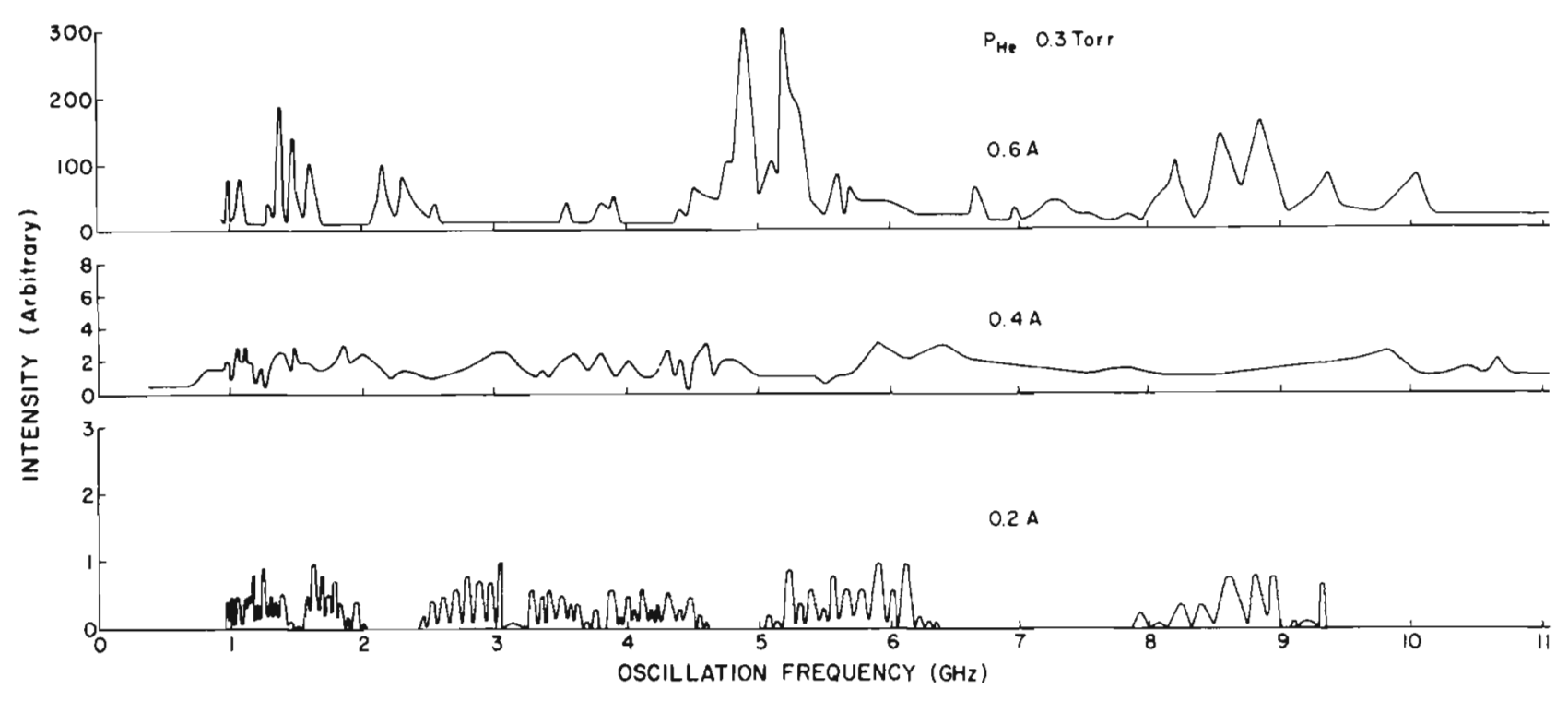

FIG. 8. Frequency spectrum of microwave signal at a pressure of 0.3 Torr and three different currents.

Figures 3 and 6 show that the current onset of these phenomena increases as the helium pressure is increased. This is in agreement with the results of Wada and Knechtli, ${ }^{13}$ who observed that the plasma density required for energy transfer from an injected electron beam to the plasma was higher when the background gas pressure was increased. This shows that collisionless thermalization of electrons in a beam-plasma system can be inhibited, if the electron collision mean-free-path is made small enough.

The spectrum of the UHF emission between 1 and 11 $\mathrm{GHz}$ was measured using an UHF spectrum analyzer, and is shown for three different discharge currents at a helium pressure of 0.3 Torr, in Fig. 8. This broad spectrum is similar to the one found in previous experiments that studied the oscillations of beam-plasma discharges, ${ }^{12,14}$ in which the dominant mode of interaction is believed to be at the plasma frequency. The plasma electrons find themselves in a if field that accelerates them to the energy range where they can excite and ionize. This increase in the excitation rate is consistent with the observed variation of the light emission. Plasma oscillations are judged to be the mechanism that efficiently transfer energy from the electron beam into the plasma, causing the observed energy degradation of the electron beam energy profile.

\section{PLASMA OSCILLATIONS IN THE EXCITATION OF CW ION LASERS}

Beam-plasma discharges, created by exciting plasma oscillations with a pulsed electron beam drifting in a longitudinal magnetic field, have been used to excite pulsed ion lasers. ${ }^{14,15}$ In these experiments an electron beam of energy between 10 and $45 \mathrm{keV}$ and current between 10 and $30 \mathrm{~A}$ was inserted into a plasma chamber, where the pressure ranged from $10^{-2}$ to $10^{-5}$ Torr. ${ }^{15}$ At this low gas density the electron beam energy would be very poorly deposited into the plasma in the absence of the collective interaction between the electron beam and the plasma, that leads to intense excitation of high-frequency oscillations.
In a cw electron beam laser system, such as the one we used to obtain cw laser action in He-metal vapor mixtures ${ }^{1.3}$ UHF oscillation could present a major disadvantage. This system is longitudinally pumped by an electron beam of approximately $3 \mathrm{keV}$. The pressure in the plasma tube is several Torr so that a beam-plasma interaction that degrades the electron energy profile in the way shown in Fig. 3 would reduce the reaching distance of the electron beam and results in a substantially shorter and nonuniform active medium.

In a transverse geometry, the range of the electron beam should be small so that the majority of the energy is deposited into the plasma and not onto the walls of the discharge chamber. Electron beam excited plasma oscillations could therefore be used to efficiently deposit the electron beam energy in the case of transverse electron beam excitation into a volume with a small cross section and long optical length. One such transverse laser excitation scheme, using glow discharge electron guns, ${ }^{2}$ is shown in Fig. 9. The anode position is not shown since its location is not essential to the characteristics of the electron beam discharge, ${ }^{2}$ nor will it block the optical path. The use of two cathodes allows for partial trap-

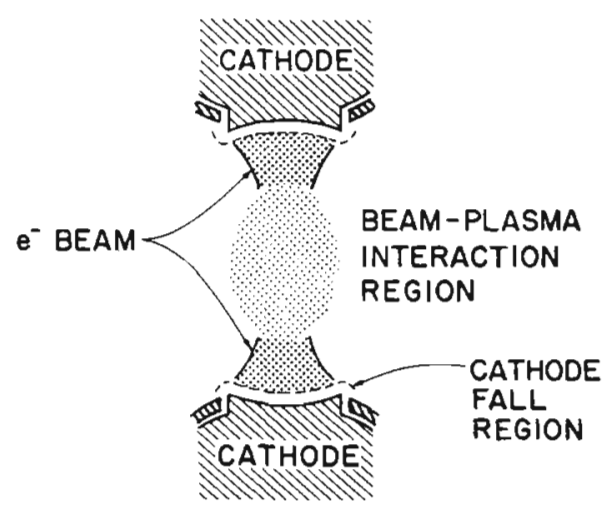

FIG. 9. Cross-sectional view of a proposed transverse electron beam pumped laser using opposing cathodes and a beam plasma interaction to achieve efficient energy deposition. 
ping of the high-energy electrons. Beam electrons which reach the opposing cathode after passing through the beamplasma region are reflected there by the electric field in the cathode fall region. Both of these effects will increase the energy deposition efficiency of the transverse electron beam.

\section{SUMMARY}

The energy spectrum of a kilovolt electron beam produced by a magnesium cathode glow discharge operating in helium at pressures between 0.15 and 0.8 Torr was measured using an electrostatic energy analyzer. The maximum energy of the electrons coincide with the discharge voltage drop, $V_{d}$. The electron beam energy spectrum measured at $17 \mathrm{~cm}$ from the electron gun presents an energy width at half-maximum of $100-300 \mathrm{eV}$. The electron beam energy spectrum gets narrower as the discharge current is incremented up to a critical current value, at which point it abruptly degrades into a broad energy profile. This change in the electron beam energy spectrum is coincident with the sudden appearance of a plasma region with a very intense luminosity and with the emission of intense microwave radiation.

This phenomena is attributed to the generation of plasma oscillations driven by the electron beam. We suggest the use of the beam-plasma interaction as a method to efficiently deposit the electron beam energy into the plasma in a transverse electron beam discharge for the excitation of ion lasers.

\section{ACKNOWLEDGMENTS}

The authors wish to thank Dr. C. E. Patton of the physics department at CSU, and Dr. R. Hunter of NBS,
Boulder, for the useful discussions, and Tom Burnell for skilled technical assistance. This work was supported by the National Science Foundation and AFOSR.

${ }^{1}$ J. J. Rocca, J. D. Meyer, and G. J. Collins, Phys. Lett. A 87, 237 (1982). ${ }^{2}$ Z. Yu, J. J. Rocca, J. D. Meyer, and G. J. Collins, J. Appl. Phys. (to be published).

${ }^{3}$ J. J. Rocca, J. D. Meyer, and G. J. Collins, Appl. Phys. Lett. 40, 300(1982). ${ }^{4}$ J. D. Meyer, J. J. Rocca, Z. Yu, and G. J. Collins, IEEE J. Quantum Electron. 18, $326(1982)$

${ }^{5}$ J. J. Rocca, J. D. Meyer, Z. Yu, and G. J. Collins, Presented at the ThirtyFourth Gaseous Electronic Conference, Boston, October 1981.

${ }^{6}$ R. M. Chaudhri and M. M. Chadhi, Proc. VIIth Int. Conf. Ion in Gases [Belgrade) 392, 1965.

${ }^{7}$ G. Carter and J. S. Colligon, Ion Bombardment of Solids (American Elsevier, New York, 1934).

${ }^{8}$ W. D. Davis and T. A. Varderslice, Phys. Rev. 131, 219 (1963).

${ }^{9}$ Comstock, Inc., Electrostatic Energy Analyzer Model AC-901 Manual, Oak Ridge, Tennessee.

${ }^{10}$ H. S. W. Massey and E. A. S. Burshop, Electronic and lonic Impact Phenomena, Vol. 1, (Oxford University Press, 1969). See also R. M. St. John, F. L. Miller, and C. C. Ling, Phys. Rev. A 134, 888 (1964)

"D. H. Looney and S. C. Brown, Phys. Rev. 93, 965 (1954).

${ }^{12}$ W. D. Getty and L. D. Smullin, J. Appl. Phys. 34, 34 (1963).

${ }^{13}$ J. Y. Wada and R. C. Knechtli, Phys. Fluids 12, 1497 (1969).

${ }^{14}$ Yu. U. Track, Ya. B. Fairberg, L. I. Bolotin, Ya. Ya. Bessarab, N. P. Gadetskii, Yu. N. Chernen'kii, and A. K. Berezin, JETP Lett. 6, 371 (1967).

${ }^{15}$ Yu. U. Track, Ya. B. Fainberg, L. I. Bolotin, Ya. Ya. Bessarab, N. P. Gadetskii, I. I. Magda, and A. V. Sidel'nikova, Sov. Phys. JETP 35, 886 (1972). 